Wilhelm Roux's Archives 189, 197-206 (1980)

\title{
Developmental Analysis of a Temperature-Sensitive Melanotic Tumor Mutant in Drosophila melanogaster
}

\author{
T.M. Rizki and Rose M. Rizki \\ Division of Biological Sciences, University of Michigan, Ann Arbor, Michigan 48109, USA
}

Summary. A sex-linked, temperature-sensitive melanotic tumor mutation in Drosophila melanogaster, $t u(1) S z^{t s}$, was mapped at $34.3 \pm$ and localized to bands $10 \mathrm{~A} 10-11$ of the polytene chromosomes. At $26^{\circ} \mathrm{C} t u-S z^{t s}$ larvae develop melanotic tumors whereas $18^{\circ} \mathrm{C}$ is non-permissive for tumor formation. Tumorigenesis at $26^{\circ} \mathrm{C}$ involves the encapsulation of abnormal caudal fat body regions by precociously differentiated hemocytes. Low temperature blocks the development of the abnormal adipose cells and the overlying aberrant tissue surfaces but does not inhibit precocious differentiation of the hemocytes to the lamellocytic form. This phenotypic difference at the two temperatures indicates that lamellocyte encapsulation to form melanotic tumors is directed against abnormal tissue surfaces. On the basis of these observations and an earlier study (Rizki and Rizki 1979) we propose that hereditary melanotic tumors in $D$. melanogaster are a class of autoimmune disorders in which affected tissue surfaces arouse the body's cellmediated defense response.

Key words: Melanotic tumors - Temperature-sensitive mutant - Cell-mediated defense - Hemocytes - Fat body - Autoimmunity.

\section{Introduction}

Melanotic tumor mutants in Drosophila melanogaster are characterized by the development of abnormal, internal black masses, generally during larval life. This phenotype is common to many nonallelic gene mutations in Drosophila but the basis for the phenotypic similarity is unknown (Sparrow 1978). One consistent feature among the melanotic tumor strains that have been examined is the participation of the larval hemocytes in the formation of the tumors. Only in the $t u-W(2,66.2 \pm$, revised) mutant, however, has the nature of the hemocyte involvement at the tumorforming site been studied in detail (Rizki and Rizki 1979). Melanotic tumor formation in $t u-W$ is restricted to the caudal fat body where the earliest visible deviation from normality is the disintegration of the basement membrane surrounding some of the adipose cells. The afflicted adipose cells are subsequently encapsulated by hemocytes to form melanized nodules that are benign. Since a similar hemocytic reaction is elicited against a variety of foreign materials entering the insect hemocoel (Salt 1970), the encapsulation reaction in $t u-W$ appears to be a defense response to rid the developing larvae of aberrant cells and tissues (Rizki 1960). If a causal relationship between afflicted tissue surfaces and hemocyte encapsulation occurs in other melanotic tumor mutants as well, it would suggest that melanotic tumor mutations are a class of genes affecting tissue surfaces in such a way that the cell-mediated defense system of the body no longer recognizes the tissues as "self". To determine whether this is the unifying feature among the group of melanotic tumor genes requires detailed analysis of the cellular components and their interactions in other melanotic tumor mutants.

We selected a previously undescribed melanotic tumor gene, $t u(I) S z^{t s}$ (abbreviated $t s$ ), for ultrastructural study of melanotic tumor formation for two reasons: (1) the site of melanotic tumors is the posterior fat body, as in $t u-W$; (2) melanotic tumors develop when $t s$ larvae are raised at $26^{\circ} \mathrm{C}$ but not at $18^{\circ} \mathrm{C}$. Therefore, the hemocytes and the fat body can be studied under tumor-permissive and tumor-restrictive conditions to analyze causal relationships between the interacting cell types during tumorigenesis. Preliminary observations on $t s$ have been summarized (Rizki and Rizki 1978). The present report includes genetic studies on this mutant and temperature shift experiments 
to determine the temperature-sensitive period for melanotic tumors as well as observations on the hemocytes and caudal fat bodies at $26^{\circ} \mathrm{C}$ and $18^{\circ} \mathrm{C}$. The following paper (in this volume) presents an experimental analysis of the factors that elicit hemocytic encapsulation in $t s$ larvae.

\section{Materials and Methods}

\section{Genetic Strains and Mapping Studies}

An EMS-induced, sex-linked melanotic tumor mutant was obtained from Dr. D. Suzuki in 1974. From this original stock we selected flies showing large tumors at $26^{\circ} \mathrm{C}$ and established a line which we designated $t u(1) S z^{t s}$ since the initial symbol used for this mutant, mel, has been preempted by the mutant melanized (Lindsley and Grell 1968). This selected line has been used for the present study, and the abbreviation $t s$ in this report refers to this line.

Drosophila mutants used in mapping studies and their sources are: Ore- $R$ wild type maintained in our laboratory; $s c c v v f B$ ras $d y$, and $D f(1) \mathrm{m}^{259-4}$ from the Bowling Green Stock Center; $D f(1) R A 37 / F M 6$ and $D f(1) N 71 / F M 6$ from Dr. G. Lefevre; $D f(1)$ $K A 7 / y$ w $f:=; D p(1: 2) v^{+65 b} /+, D f(1) R A 37 / F M 7 c$, and $D F(1)$ $v^{L 3} / y w f:=; D p(1: 2) v^{+63 i} /+$ from the Stock Center at the California Institute of Technology (for terminology and symbols see Lindsley and Grell 1968). Each of the stocks crossed to $t s$ was checked to verify that it did not develop melanotic tumors. under the conditions used for the given experiment in which the stock was utilized.

Crosses for locating the $t s$ gene were made in culture bottles containing standard corn meal-molasses medium and maintained in an incubator fluctuating between $26^{\circ}-27^{\circ} \mathrm{C}$. Offspring from the matings of $t s$ flies carrying duplications and/or deficiencies were grown at $26^{\circ}-27^{\circ} \mathrm{C}$ in crystallizing dishes containing cream of wheat medium. Crowding was avoided by placing $80-100$ larvae in each $90 \mathrm{~mm}$-diameter dish.

\section{Determination of the Temperature-Sensitive Period}

For "shift-up" experiments larvae were collected within $2 \mathrm{~h}$ after hatching at $18^{\circ} \mathrm{C}$ and placed in half-pint bottles containing corn meal-molasses medium seeded with live yeast. Two bottles $(60$ larvae each) were transferred to a $26^{\circ} \mathrm{C}$ incubator every eight hours until pupariation at approximately $176 \mathrm{~h}$. White puparia and early tan puparia were separately isolated at this time and transferred to the higher temperature. Melanotic masses were scored by dissecting the adult flies. "Shift-down" experiments followed the same procedure except that larvae were collected at $26^{\circ} \mathrm{C}$ and transferred to $18^{\circ} \mathrm{C}$ every six hours.

\section{Hemocyte Examination}

Newly hatched larvae were transferred to cream of wheat-molasses medium in crystallizing dishes kept in incubators fluctuating between $18^{\circ}-19^{\circ} \mathrm{C}$ or $26^{\circ}-27^{\circ} \mathrm{C}$. Hemolymph samples were obtained from timed larvae by opening the body wall under a drop of mineral oil. The remainder of the carcass was removed quickly, taking care not to rupture internal organs. A coverglass was then gently placed on the oil droplet and the samples were examined immediately with phase optics.

\section{Electron Microscopy}

Posterior fat bodies and regions of the posterior body walls of timed larvae growing on cream of wheat medium were fixed in $2.3 \%$ formaldehyde in phosphate buffer at $\mathrm{pH} 7.15$. After a buffer rinse, the tissues were postfixed in buffered osmium for $20 \mathrm{~min}$. Specimens for scanning electron microscopy (SEM) were dehydrated through a graded series of alcohols, amyl acetate, and processed through critical point drying. They were mounted on aluminum stubs, sputter-coated with gold, and examined in a JEOL-U3 SEM. Specimens for transmission electron microscopy (TEM) were dehydrated through a graded series of alcohols, transferred to propylene oxide, and embedded in epon. Thin sections were stained with Reynold's lead citrate and uranyl acetate, and examined in a Philips 300 TEM.

\section{Results}

\section{Mapping the tu(1)Sz Locus}

Experiment 1 . Heterozygous females from the cross, ts females X sc $c v$ of $B$ males, were crossed to Ore- $R$ males. The progeny males were classified according to the external markers and subsequently dissected to confirm the presence or absence of melanotic tumors. A total of 664 males was sorted out. The classes of males generated by single exchanges between intervals defined by the sc $c v$ v f $B$ markers indicated that the $t s$ gene is located to the right of the $v^{+}$ locus. Analysis of the pooled data segregating from the $v+f l+t s+$ females showed a $v-t s$ recombination value of $1.2 \pm 0.4$. Therefore, subsequent crosses utilized markers located within two crossing over units of this estimated position $(34 \pm)$ of the $t s$ gene.

Experiment 2. Flies with the markers ras $d y$ were crossed to $t s$ and the $\mathrm{F}_{1}$ females mated with $t s$ males. The testcross generation males were first classified according to eye color and wing character and then each male was dissected to determine the tumor phenotype. From a total of 649 males, the recombination value between ras - ts was 1.54 and between $t s$ $d y$ was 1.69 . The sum of the two values is $3.23 \pm 0.69$ (expected: ras $-d y=3.4$, Lindsley and Grell 1968).

There were 10 exchanges between ras and $t s$, and 11 between $t s$ and $d y$. If the standard value for the ras - $d y$ interval, 3.4 , is proportioned on the basis of these exchanges $(10 / 21$ and $11 / 21)$, then the estimate for the ras - ts interval is 1.62 and for $t s-$ $d y$ is 1.78 .

The values for the distances between the markers and $t s$ are summarized:

$\begin{array}{llll}\text { Experiment 1: } & v & 1.2 & t s \\ \text { Experiment 2: } & \text { ras } & 1.54 & t s \\ \text { Estimate: } & \text { ras } & 1.62 & t s\end{array}$




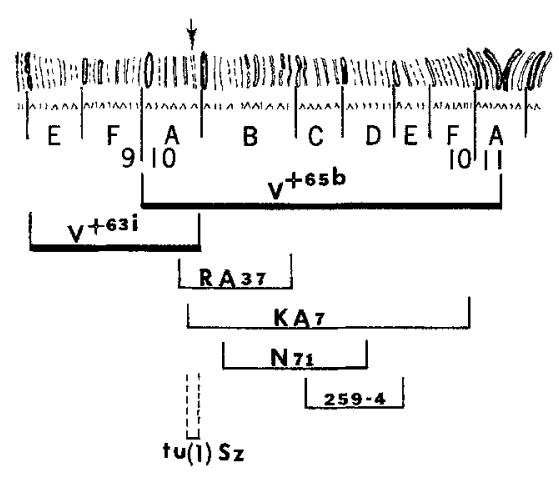

Fig. 1. Diagrammatic representation of the $X$ chromosome (Lindsley and Grell 1968) region $9 \mathrm{E}$ to $11 \mathrm{~A}$ showing the extent of the overlapping duplications (heavy lines) and deficiencies (thin lines) used for assignment of the $t u(1) S z^{+}$locus to the doublet 10A10-11 (indicated by the arrow)

This information provides the following assignment for the $t u(1) S z^{+}$locus:

$\begin{array}{llll}\text { ras } & v & t s & d y \\ 32.8 & 33 & 34.2 & 36.2 \\ & & 34.34 & \\ & & 34.42 & \\ & & 34.3 \pm & \end{array}$

Crosses were then made between $t s$ and $\mathrm{X}$ chromosomes with overlapping deficiencies to the right of the $v$ locus: $D f(1) R A 37, D f(1) K A 7, D f(1) N 71$, and $D f(1) \mathrm{m}^{259-4}$. The heterozygous $t s / D f$ females from these matings were examined for the presence of tumors at $26^{\circ}$ C. $D f(1) R A 37$ and $D f(1) K A 7$ were noncomplementary whereas $t s$ was complemented by chromosomes carrying deficiencies $N 71$ and $m^{259-4}$. According to Dr. G. Lefevre (personal communication) $D f(1) R A 37$ includes salivary gland chromosome bands $10 \mathrm{~A} 8$ to $10 \mathrm{~B} 17, D f(1) K A 7$ includes bands $10 \mathrm{~A} 10$ to $10 \mathrm{~F} 10$, and $D f(1) N 71$ includes bands $10 \mathrm{~B} 5$ to 10D4. $D f(1) m^{259-4}$ extends from $10 \mathrm{C} 2-3$ to $10 \mathrm{E} 2-3$ (Lindsley and Grell 1968). Therefore, the $t_{s}$ locus must reside within bands 10A10-10B4.

The $t s$ mutant was further tested against two duplications $(1 \rightarrow 2): D p v^{+65 b}$ and $D p v^{+63 i}$. Both complemented $t s$, therefore $t s$ must be included in the regions covered by these duplications. $D p v^{+65 b}$ extends from 10A1 to $11 \mathrm{~A} 7$ and $D p v^{+63 i}$ includes bands 9E1 and overlaps $v^{+65 b}$ to 10A11 (Lefevre 1969). Since the doublet $10 \mathrm{~A} 10-11$ is missing in $K A 7$ which is non-complementary for $t s$ and this doublet is included in the duplication $63 i$ which is complementary, $t s$ must be located within these two bands. This information is summarized in Fig. 1.

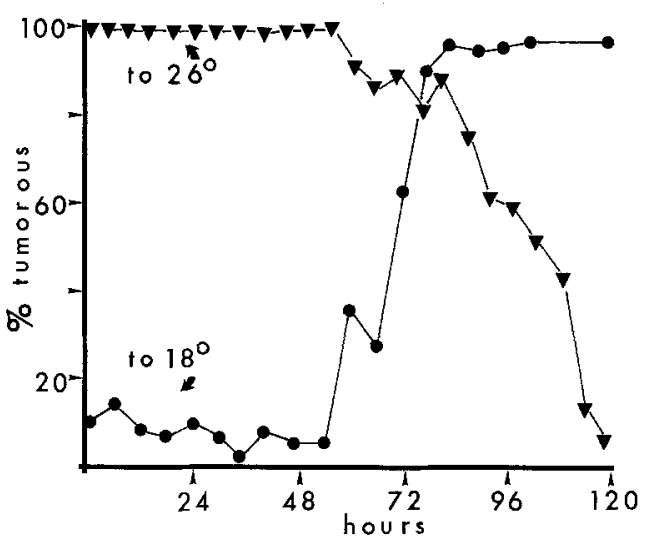

Fig. 2. Results of temperature shift studies in which $t s$ larvae were transferred from $18^{\circ} \mathrm{C}$ to $26^{\circ} \mathrm{C}$, and vice versa. Since the larval period is approximately doubled at $18^{\circ} \mathrm{C}$, the data have been normalized to a common time scale representing hours from egg hatching; the third instar begins at approximately $46 \mathrm{~h}$. Adult flies were scored as percent tumorous

Table 1. Early third instar larvae with precocious lamellocytes

\begin{tabular}{|c|c|c|}
\hline \multirow[t]{2}{*}{ Genotype } & \multicolumn{2}{|l|}{ Temperature } \\
\hline & $18^{\circ} \mathrm{C}$ & $26^{\circ} \mathrm{C}$ \\
\hline$+1+($ Ore $-R)$ & $0 \%(10)^{a}$ & $0 \%(10)$ \\
\hline $\mathrm{ts} / \mathrm{ts}$ & $100 \%(10)$ & $100 \%(10)$ \\
\hline $\mathrm{ts} / \widehat{\phi}$ & $100 \%(10)$ & $100 \%(10)$ \\
\hline \multicolumn{3}{|l|}{ Cross $+1+\mathrm{X}$ ts $/ \mathrm{o}^{\pi}:$} \\
\hline ts $/+$ & $100 \%(10)$ & $100 \%(14)$ \\
\hline+10 & $0 \%(10)$ & $0 \%(11)$ \\
\hline
\end{tabular}

a (Sample Size)

\section{Temperature-Sensitive Period}

The temperature-sensitive period for melanotic tumors was determined by shifting larvae growing at $18^{\circ} \mathrm{C}$ to $26^{\circ} \mathrm{C}$ and other groups of larvae from $26^{\circ} \mathrm{C}$ to $18^{\circ} \mathrm{C}$ at various times during development. Control groups were maintained at the two temperatures throughout their development. Melanotic tumors were scored in the adults hatching from the cultures. In classifying an internal black mass as a melanotic tumor, size was not considered. It should be noted, however, that a low incidence $(2 \%-3 \%)$ of minute melanotic specks was recorded in adult controls at $18^{\circ} \mathrm{C}$ whereas controls raised at high temperature generally had large melanotic masses. The results for the males and females were similar so the sets of data were pooled and shift times standardized to a common scale (Fig. 2).

The temperature-sensitive period for melanotic tumors coincides with the third larval instar. There is not a clear-cut, limited time interval during which 

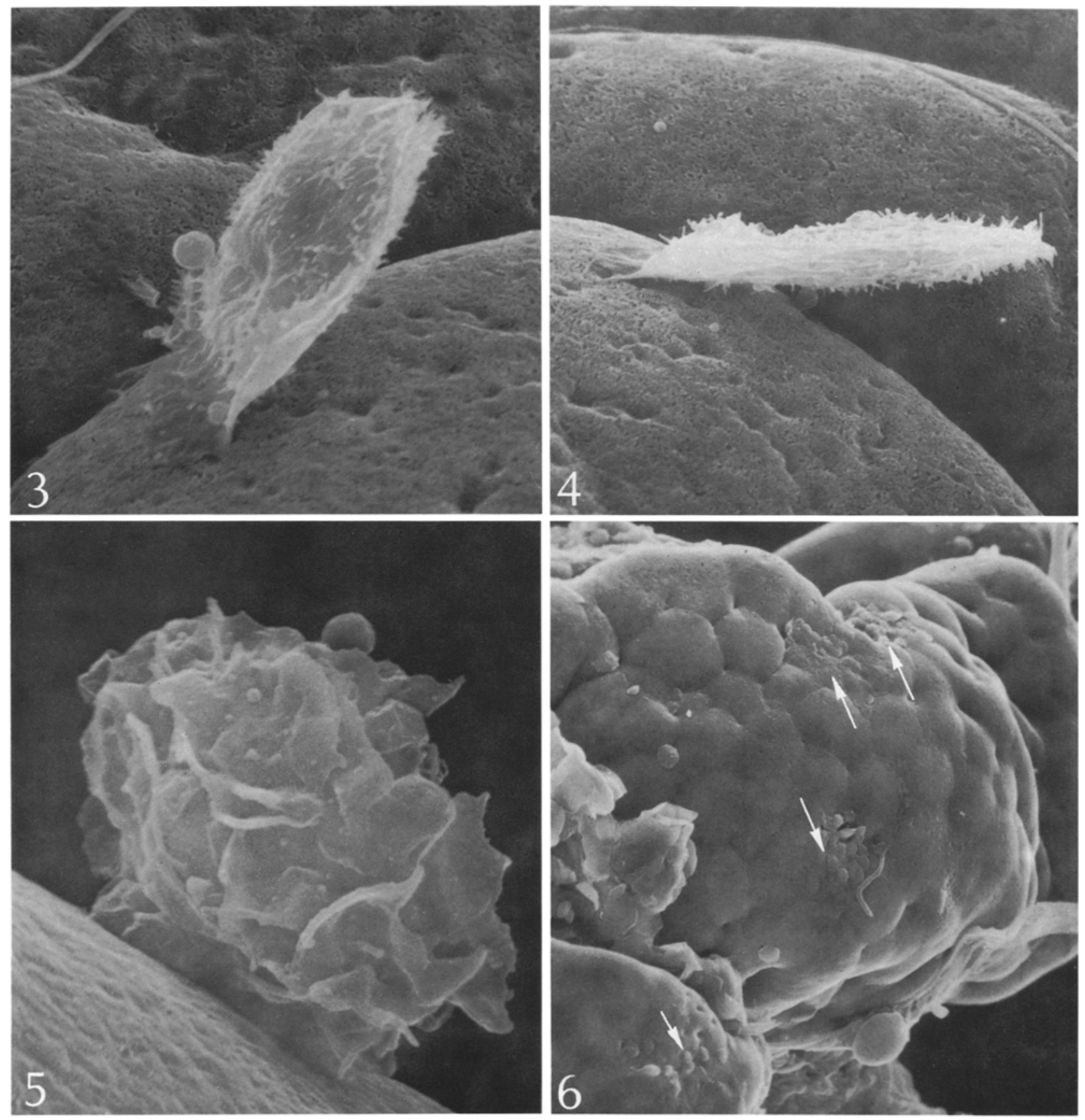

Figs. 3 and 4. Two views of a lamellocyte to illustrate cell shape and surface features. This cell in a $t s$ larva at $26^{\circ} \mathrm{C}$ is not fully flattened; note the presence of blebs and the highly filamentous surfaces. $\times 3,400$

Fig. 5. A highly ruffled surface of a hemocyte presumably in the early stage of differentiation to the lamellocytic form; $t s$ larva at $26^{\circ}$ C. $\times 6,700$

Fig. 6. Aggregates of hemocytes (arrows) at different sites on the caudal fat body surface of a ts larva at $26^{\circ} \mathrm{C} . \times 215$

melanotic tumor formation is prevented or released by a shift-down or shift-up in temperature. Rather, a progressively larger percentage of flies with melanotic tumors results as the time spent at the higher temperature is increased. Maximum penetrance and expressivity is obtained by keeping the larvae at $26^{\circ} \mathrm{C}$ for the first half of the third instar, and shift-down after this point does not affect the outcome. Furthermore, approximately $50 \%$ of the maximum frequency is reached within the first twelve hours of the third 

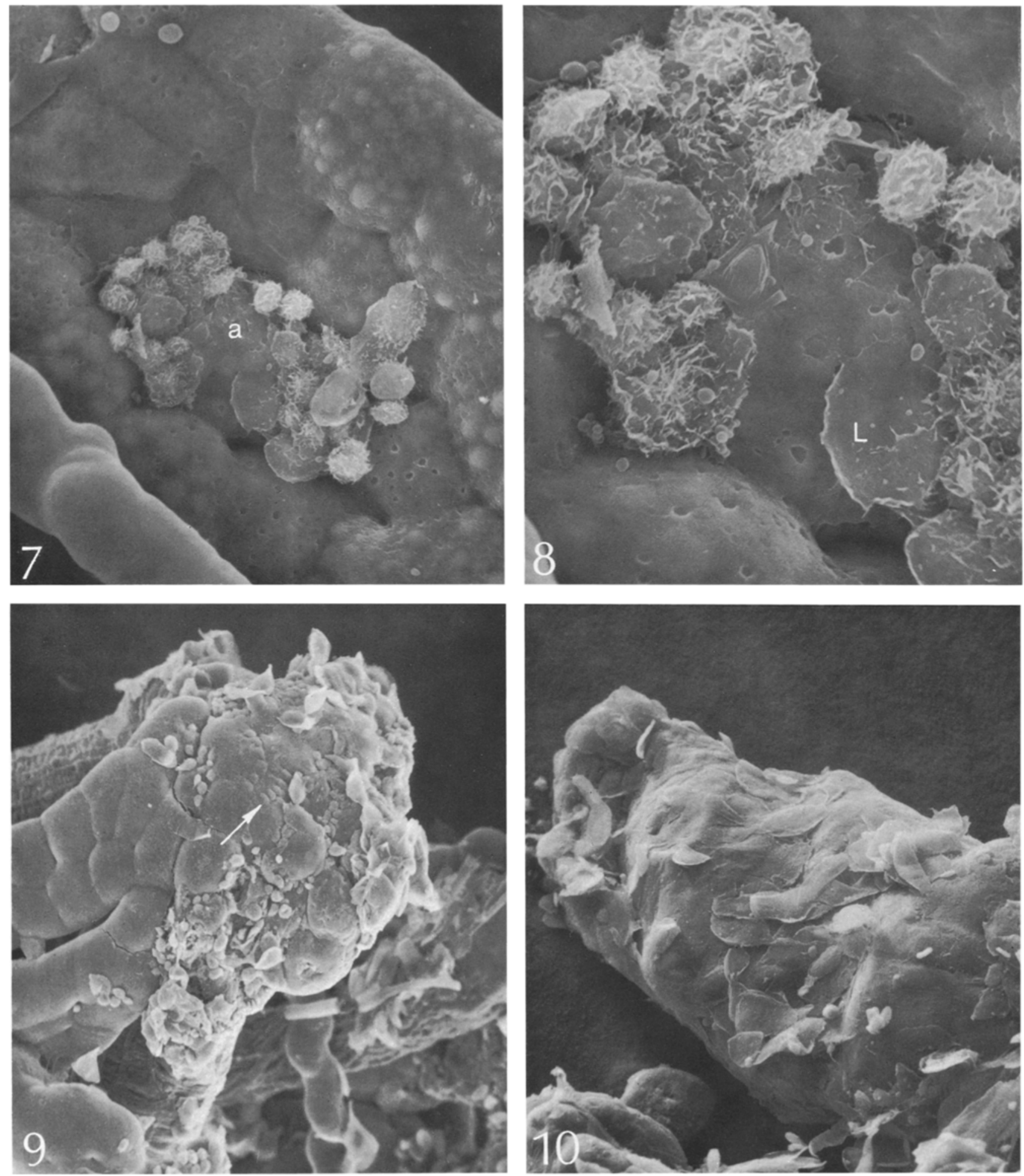

Fig. 7. Aggregation of hemocytes at the margins of a single adipose cell, $a$, surrounded by adipose cells without hemocytes on their surfaces. $\times 550$

Fig. 8. The same group of hemocytes as in Fig. 7. Note their highly filamentous and ruffled surfaces and a fully differentiated lamellocyte, L. $\times 1,360$

Fig. 9. An advanced stage of tumorigenesis showing the accumulation of lamellocytes and infiltration of hemocytes through the surface covering of the tissue (arrow). $\times 200$

Fig. 10. A melanotic tumor illustrating the smoothness of the fully differentiated lamellocytes covering the caudal fat body. $\times 200$ 


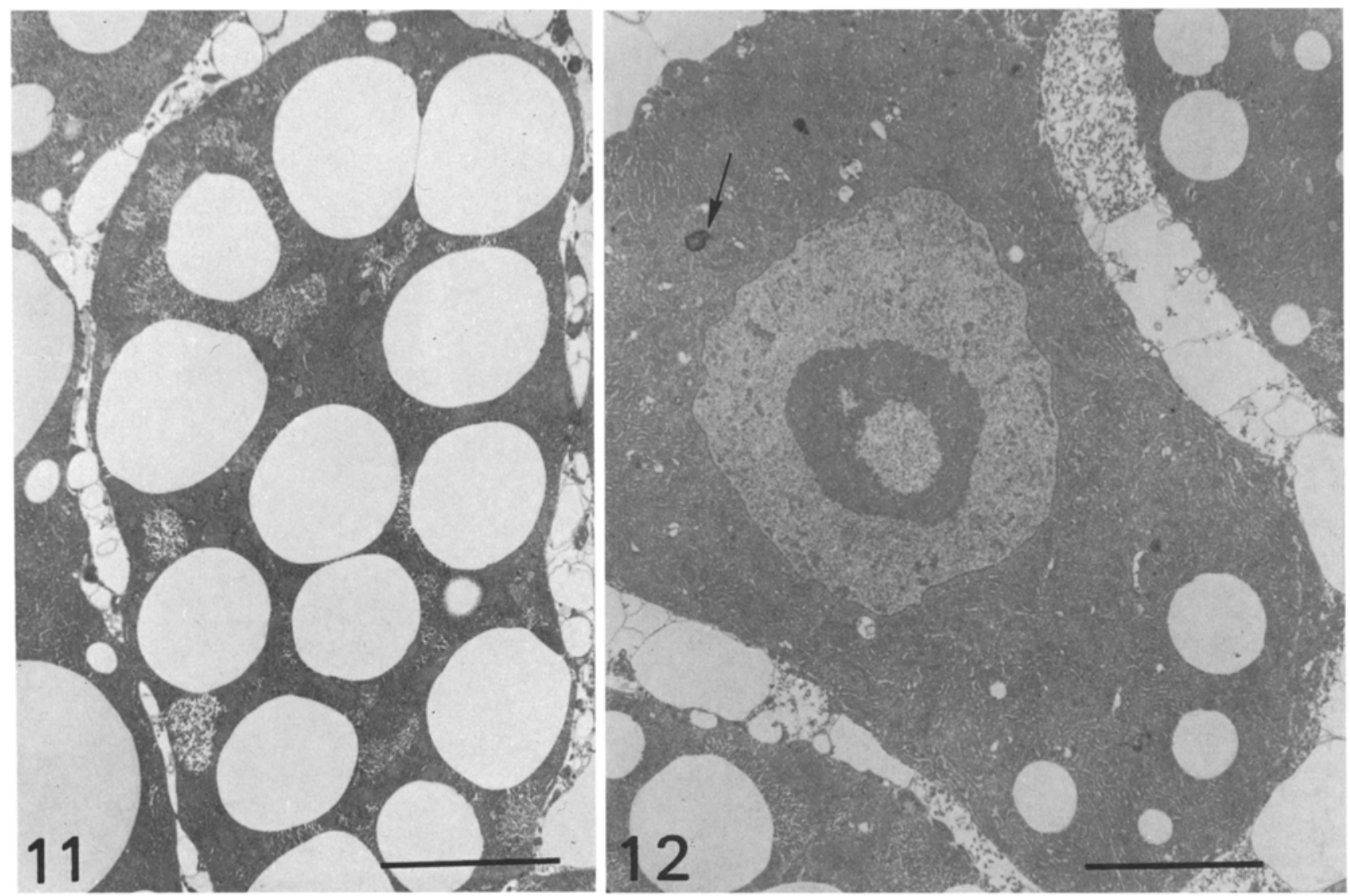

Fig. 11. Section through a cell from the caudal fat body of a $t s$ larva at $26^{\circ} \mathrm{C}$ shortly after the second molt. This is the characteristic appearance of normal fat body cells of Drosophila. Scale $=5 \mu \mathrm{m}$

Fig. 12. A hypolipidic fat body cell adjacent to the cell in Fig. 11. The arrow points to a membranous whorl. Scale $=5 \mu \mathrm{m}$

instar at this temperature. On the other hand, inhibition of melanotic tumors equivalent to the control value at $18^{\circ} \mathrm{C}$ is not attained until pupariation, and the $50 \%$ level is not reached until shortly before this point.

\section{Larval Hemocytes}

Melanotic tumor strains are characterized by a precocious presence of lamellocytes in the larval hemocoel (Rizki 1957a, 1978). In the Ore- $R$ wild type these flattened variants of the plasmatocytes do not appear in large numbers until the end of larval life (Rizki $1957 \mathrm{~b}$ ). The early appearance of lamellocytes in melanotic tumor mutants is significant since these flattened hemocytes form the multilayered walls of the tumors. As expected, early third instar $t s$ larvae growing at the tumor-permissive temperature contain many lamellocytes. However, lamellocytes are equally prominent in $t s$ larvae at $18^{\circ} \mathrm{C}$ indicating that early hemocyte transformation in $t s$ is not a temperaturesensitive trait.
To examine the "lamellocyte effect" of the $t s$ syndrome, progeny from a cross of Ore- $R$ females to $t s$ males were examined. For this study larvae growing at $18^{\circ} \mathrm{C}$ and $26^{\circ} \mathrm{C}$ were sexed prior to taking hemolymph samples. The data are summarized in Table 1 together with information on the hemocytes from control groups of Ore- $R$ and $t s$ larvae. None of the male larvae from the cross showed the precocious presence of lamellocytes whereas every female larva did regardless of the temperature. Therefore, the precocious presence of lamellocytes in $t s$ is dominant as well as temperature independent.

\section{Melanotic Tumor Formation}

The presence of lamellocytes in third instar $t s$ larvae is confirmed by SEM examination (Figs. 3 and 4), and many of the plasmatocyte surfaces are highly ruffled (Fig. 5) or filamentous. The first sign of tumorigenesis at $26^{\circ} \mathrm{C}$ is the presence of hemocytes on the surfaces of the posterior fat body in the region 

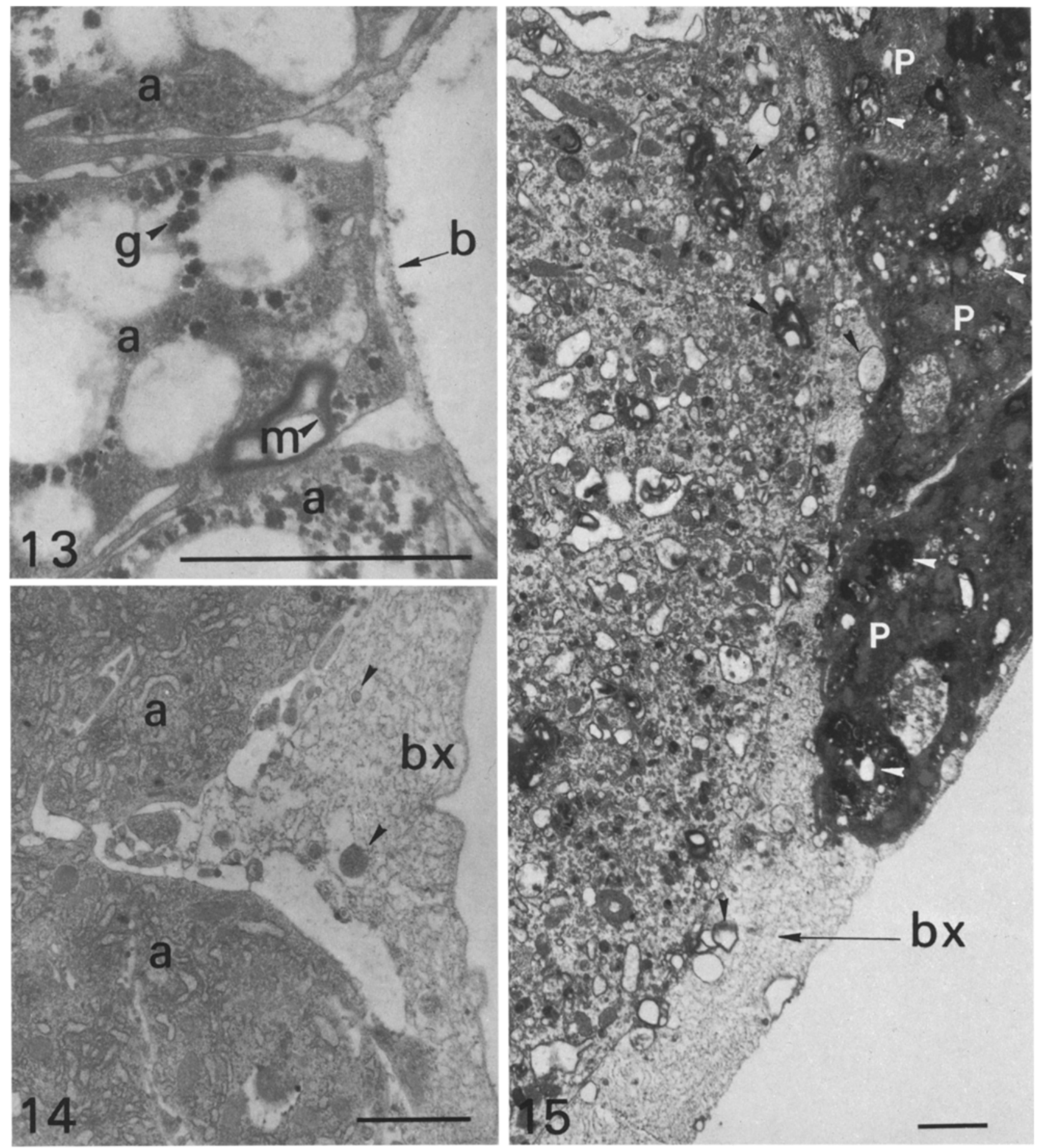

Fig. 13. Section through the periphery of adipose cells, $a$, of a Ore- $R$ region five fat body showing the basement membrane, $b$, lipid droplets, glycogen, $g$, and a membranous whorl, $m$. Scale $=1 \mu \mathrm{m}$

Fig. 14. The peripheral region of two adipose cells, $a$, of a ts larva at $26^{\circ} \mathrm{C}$. The unusual accumulation of basement membranelike material, $b x$, contains electron dense inclusions (arrowheads). Note the absence of lipid droplets and the extensive ER. Scale $=1 \mu \mathrm{m}$

Fig. 15. Infiltration of hemocytes, $p$, in the basement membranelike matrix, $b x$, at the periphery of a hypolipidic cell. Membranous inclusions from the adipose cell have been extruded into the matrix (black arrowheads) and the presence of similar elements (white arrowheads) within the hemocytes indicates that these materials are phagocytized by the blood cells. Scale $=1 \mu \mathrm{m}$ 

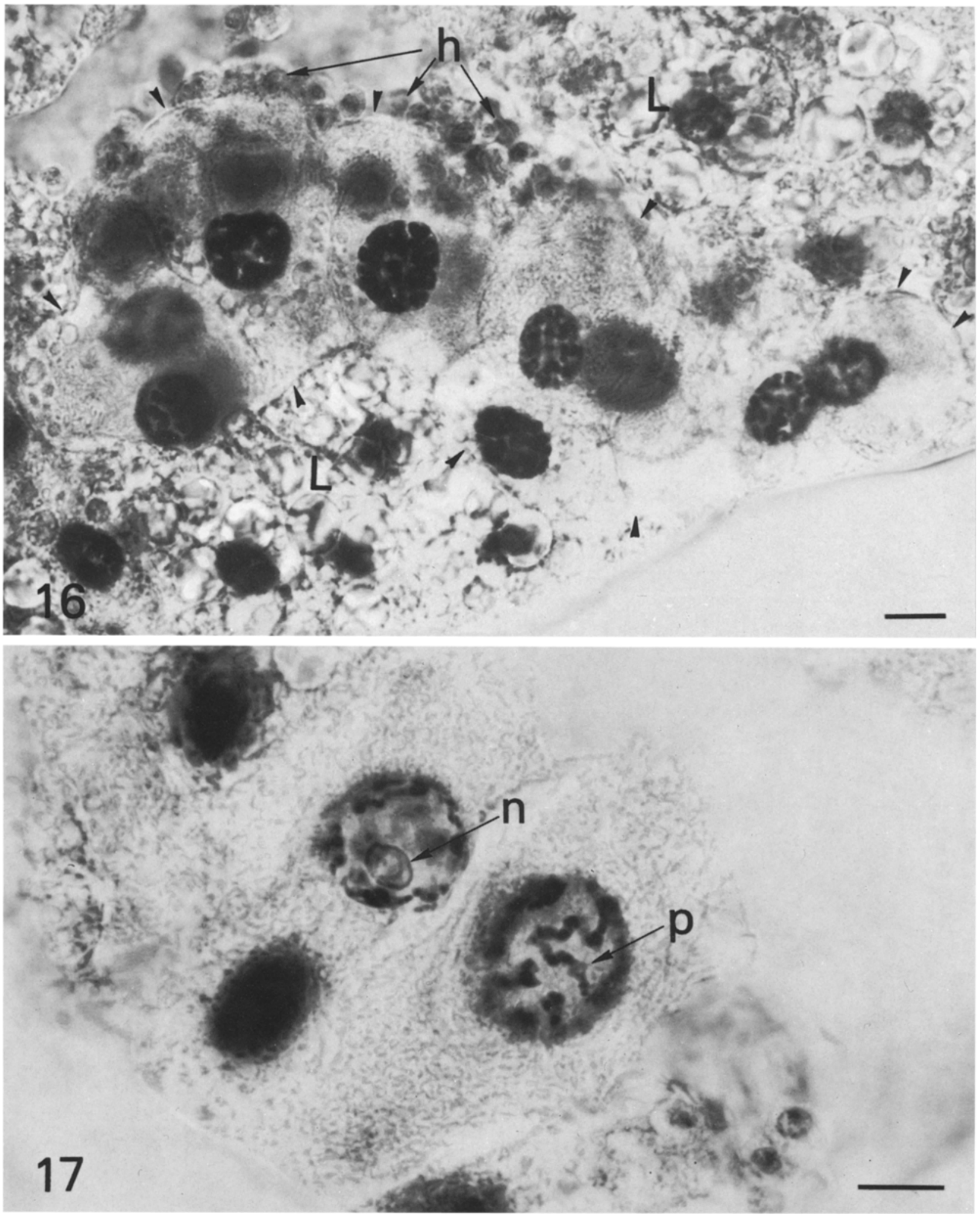

Fig. 16. Feulgen whole-mount preparation of $t s$ adipose tissue region five $\left(26^{\circ} \mathrm{C}\right)$. Arrowheads indicate a group of 13 hypolipidic cells; 6 nuclei are in the focal plane of the objective. These nuclei are larger than the nuclei of the lipid-containing cells, $L$. Hemocytes, $h$, are gathered at the periphery of the hypolipidic cells. The substage condenser diaphragm has been stopped down to bring out the outlines of the cells and lipid droplets in this and the following photograph. Scale $=10 \mu \mathrm{m}$

Fig. 17. Nuclei of two hypolipidic cells, one showing a polytene chromosome with a prominent puff, $p$. The other shows the outline of the nucleolus, $n$. Scale $=10 \mu \mathrm{m}$ 
designated "five" (Rizki 1964). That this hemocyte adherence is specific and not a random process is clear since hemocyte aggregation occurs at the surfaces of individual adipose cells or a few adipose cells that have a discontinuous distribution among the cells of region five fat body (Figs. 6 and 7). The hemocytes aggregating in these areas show many ruffles, filaments, and large globules on their surfaces (Fig. 8); lamellocytes are present among the aggregations. As hemocyte accumulation continues at the posterior fat body, larger areas of the adipose tissue become covered (Fig. 9). In some specimens the hemocytes infiltrate the surface layer of the tissue whereas other hemocytes are clearly spread over the surfaces of the fat body.

Epon sections of posterior fat bodies of $t s$ larvae fixed immediately following the second molt at $26^{\circ} \mathrm{C}$ show two types of adipose cells. Some adipose cells are normal and full of lipid droplets similar to those seen in Ore- $R$ specimens whereas other $t s$ adipose cells are markedly hypolipidic (Figs. 11 and 12). Such atypical adipose cells occur individually or as groups of two or more cells among cells with normal lipid droplets, giving the tissue region a mosaic appearance. Fibrous materials subsequently accumulate beneath the basement membrane covering the hypolipidic cells (Compare basement membrane, b, in Fig. 13 with bx in Fig. 14). The nature of this extracellular accumulation is unclear and requires further study, however, in some regions this material contains vesicular membranous profiles or particulate substances that apparently originate by fragmentation of the peripheral cytoplasm. Membranous whorls are occasionally found in late third instar Ore-R fat bodies (Fig. 13) but whether these and the variety of myelin forms accumulating in $t s$ hypolipidic cells have a similar origin requires further study. Hemocytes infiltrate the aberrant basement membrane of $t s$ fat bodies and actively phagocytize material dispersed within the membranous matrix (Fig. 15, which corresponds to SEM Fig. 9).

The specificity of hemocyte accumulation on the surfaces of the hypolipidic cells can also be seen in whole-mount preparations of Feulgen-stained fat bodies (Fig. 16). This method of examination reveals an additional difference between the hypolipidic and normal adipose cells. The nuclei of the hypolipidic cells are larger and their polytene chromosomes show prominent bands and puffs (Fig. 17). Detailed studies on these nuclear changes are in progress.

In fully formed cellular capsules all of the hemocytes at the fat body surfaces are in the lamellocytic form (Fig. 10). Melanization of the cellular capsules generally occurs toward the end of the larval period. Sections through such capsules show the same layer- ing of lamellocytes as published photographs of $t u-W$ melanotic tumors (Rizki and Rizki 1979).

Massive accumulation of hemocytes as seen at $26^{\circ} \mathrm{C}$ was not found in $t s$ specimens growing at $18^{\circ} \mathrm{C}$. When these specimens were examined by SEM blood cells were found on the fat body surfaces of several larvae. In these cases the hemocyte activity was restricted to the surfaces of one or two adipose cells. This limited hemocyte activity may account for the low incidence of minute melanotic specks noticed among flies raised at this temperature. We did not find any fully developed melanotic tumors in samples of $t s$ larvae fixed for SEM, TEM, and histological examination. To assure that the hemocyte adherence in ts larvae at $18^{\circ} \mathrm{C}$ was a feature of the mutant and not a random distribution of hemocytes due to fixation artifact, we reexamined fat bodies of control groups of Ore- $R$ larvae. Hemocytes were not found on the Ore- $R$ fat body surfaces, confirming observations made in earlier studies (Rizki and Rizki 1974, 1979). No hypolipidic cells or abnormal tissue surfaces were found in paraffin or thin epon sections of fat bodies from $t s$ larvae raised at $18^{\circ} \mathrm{C}$.

\section{Discussion}

The importance of tissue surface changes in initiating the encapsulation activity of the hemocytes is clearly apparent in $t s$. Failure to develop encapsulated masses at $18^{\circ} \mathrm{C}$ is correlated with the absence of the tumorforming site, i.e., the atypical adipose cells, and not the availa bility of lamellocytes. Furthermore, at $26^{\circ} \mathrm{C}$ hypolipidic cells and lamellocytes are present at the beginning of the third larval instar, but the encapsulation process does not occur until changes at the peripheral regions of the hypolipidic cells and the basement membrane become noticeable. Therefore, the effect of the shift down in temperature is to eliminate the tumor-forming site by partially normalizing the adipose cells rather than suppressing the entire $t s$ syndrome. The hypolipidic condition at high temperature suggests that a biochemical lesion in lipid metabolism underlies melanotic tumor formation in this mutant. Whether this effect represents faulty synthesis or improper lipid storage is not known. However, these effects on lipid metabolism probably influence the thermal stability of intracellular membranes and may account for the aberrant changes in the cytoplasmic contents and accumulations at the cell surfaces. It is not known whether the primary effect on lipid metabolism is limited to the adipose cells; nor is there an apparent explanation for the mosaic distribution of the hypolipidic cells within the fat body. 
The early appearance of lamellocytes in the larval hemolymph at $26^{\circ} \mathrm{C}$ is recognized as a part of the melanotic tumor syndrome in $t s$ larvae. However, the presence of lamellocytes in the larval hemolymph at $18^{\circ} \mathrm{C}$ and as a dominant phenotype in heterozygotes at both $18^{\circ} \mathrm{C}$ and $26^{\circ} \mathrm{C}$ requires an explanation. Since the precocious presence of lamellocytes in larval life is a part of the common syndrome of nonallelic melanotic tumorous strains of Drosophila and the lamellocytic blood cell shape is crucial for the formation of the melanotic tumor walls, it is likely that the earliest deviations in melanotic tumor mutant larvae stimulate the changes in hemocyte morphology. We cannot, however, exclude the possibility that the hemocyte effects in $t s$ result from a tightly-linked dominant gene; evidence for this requires specific markers (protein or enzyme) for each of the cellular effects (hemocyte transformation and tumor-forming site) so that recombinants between the two gene loci can be recognized in the absence of melanotic tumor formation (epigenetic phenotype). The SEM observations of minor hemocyte adherence to fat body surfaces of some specimens growing at $18^{\circ} \mathrm{C}$ suggest that the difference in the $t s$ mutant syndromes at the two temperatures is quantitative rather than a qualitative distinction at the two temperatures. Even though all the adipose cells in $t s$ larvae at $18^{\circ} \mathrm{C}$ contain lipid droplets, some of these cells must not be functioning in a completely normal fashion; i.e., lowering the temperature for larval growth minimizes the tissue abnormality but does not eliminate it. We did not examine fat bodies of heterozygotes; the presence of lamellocytes in this case may indicate that the mutant gene in one dose also generates a partial effect to the extent that the hemocytes are affected.

The $t s$ syndrome includes events described for the nonallelic melanotic tumor mutant, $t u$ - $W$ : precocious presence of lamellocytes in the early third instar, phagocytosis of particulate materials escaping from adipose cells, encapsulation of adipose cells by lamellocytes to form cellular capsules, and melanization (Rizki and Rizki 1978, 1979). Abnormalities at tissue surfaces precede the encapsulation phenomenon in both mutants but the details of the changes at the fat body surfaces differ. The $t s$ and $t u-W$ loci must specify intracellular changes in the fat body that are distinct in each case. Thus, melanotic tumor formation may be divided into two processes: (1) a gene-conditioned developmental upset in specific cell types or tissues; (2) hemocyte responses to the aberrant cells or tissues. The latter phenomenon encompassing the body's cellmediated defense system is common to all melanotic tumor strains that have been examined (Oftedal 1953; Rizki 1957a; Sang and Burnet 1963), suggesting that the common denominator among these mutants is the arousal of the body's defense system. On the basis of the studies with $t s$ and $t u-W$ we suggest that modification at tissue surfaces is the critical feature for initiating the encapsulation response, and melanotic tumor mutants are a class of autoimmune disorders in Drosophila.

Acknowledgements. We are grateful to Dr. D. Suzuki (University of British Columbia), Dr. G. Lefevre, Jr. (California State University, Northridge), Dr. R.C. Woodruff and P. Oster (Bowling Green State University Stock Center), and Dr. L. Cramer (California Institute of Technology Stock Center) for providing the Drosophila stocks used in this study. This investigation was supported by NIH Grant No. CA 16619

\section{References}

Lefevre $G$ Ir (1969) The eccentricity of vermilion deficiencis in D. melanogaster. Genetics 63:589-600

Lindsley DL, Grell EH (1968) Genetic Variations of Drosophila melanogaster. Carnegie Inst Washington Publ 627

Oftedal P (1953) The histogenesis of a new tumor in $D$. melanogaster and a comparison with tumors of five other stocks. Z. Indukt Abstamm Vererbungsl 85:408-422

Rizki RM, Rizki TM (1974) Basement membrane abnormalities in melanotic tumor formation of Drosophila. Experientia $30: 543-546$

Rizki RM, Rizki TM (1979) Cell interactions in the differentiation of a melanotic tumor in Drosophila. Differentiation 12:167-178

Rizki TM (1957a) Tumor formation in relation to metamorphosis in Drosophila melanogasier. J Morphol 100:459-472

Rizki TM (1957b) Alterations in the haemocyte population of Drosophila melanogaster. J Morphol 100:437-458

Rizki TM (1960) Melanotic tumor formation in. Drosophila. J Morphol 106:147-158

Rizki TM (1964) Mutant genes regulating the inducibility of kynurenine synthesis. J Cell Biol 21:203-211

Rizki TM (1978) The circulatory system and associated cells and tissues. In: Ashburner M, Wright TRF (eds) The genetics and biology of Drosophila, Vol 2b. Academic Press, London pp $397-$ 452

Rizki TM, Rizki RM (1978) The role of hemocytes in melanotic tumor formation. In: Bulla LA, Cheng TC (eds) Comparative pathobiology, vol. 4. Plenum Press, New York pp 85-96

Salt $\mathrm{G}(1970)$ The cellular defense reactions of insects. University Press, Cambridge

Sang JH, Burnet B (1963) Physiological genetics of melanotic tumors in Drosophila melanogaster. I. The effects of nutrient balance on tumor penetrance in the $t u-k$ strain. Genetics $48: 235$ 253

Sparrow JC (1978) Melanotic tumours. In: Ashburner M, Wright TRF (eds) The genetics and biology of Drosophila, vol $2 \mathrm{~b}$. Academic Press, London pp 277-313

Received May 2, 1980

Accepted in revised form August 27, 1980 